\title{
Colloid Carcinoma of the Uterine Cervix and Its Immunohistochemical Analysis: A Case Report
}

\author{
Nermin Koc · Sevcan Arzu Arinkan \\ Nurver Ozel Ozbay ${ }^{2}$. Selcuk Selcuk ${ }^{1}$ \\ Departments of Pathology and ${ }^{1}$ Obstetrics and \\ Gynecology, Zeynep Kamil Maternity and \\ Pediatric Research and Training Hospital, Istanbul; \\ ${ }^{2}$ Department of Pathology, Fatih Sultan Mehmet \\ Research and Training Hospital, \\ Istanbul, Turkey
}

Received: September 29, 2016

Revised: March 27, 2017

Accepted: April 8, 2017

\section{Corresponding Author}

Sevcan Arzu Arinkan, MD

Department of Obstetrics and Gynecology, Zeynep

Kamil Maternity and Pediatric Research and

Training Hospital, Istanbul 34660, Turkey

Tel: +90-505-683-7557

Fax: +90-216-391-0680

E-mail: pataraa96@gmail.com
Colloid carcinoma, which is a very rare tumor of the uterine cervix, is composed of an excessive amount of mucus and a relative paucity of tumoral glandular cells within them. Herein, we report a rare case of colloid carcinoma of the cervix with adenocarcinoma in situ (AIS), intestinal and usual types, and endocervical adenocarcinoma (usual type) components. We also discuss the morphological and immunohistochemical characteristics of this tumor. A 51-year-old woman was referred to our outpatient clinic with the symptom of genital bleeding lasting for 5 months. She had a cervix surrounded by an irregular tumor with a diameter of $5 \mathrm{~cm}$. The colloid carcinoma cells were positive for MUC2, MUC5AC, and cytokeratin (CK) 7, focal positive for CDX2, and negative for MUC6 and CK20. Also, the intestinal type AIS showed a similar staining pattern. Colloid carcinoma cells producing mucin showed an intestinal phenotype and AIS. The intestinal type can be considered as a precursor lesion of colloid carcinoma.

Key Words: Colloid carcinoma; Cervix uteri; Neoplasms
Colloid carcinoma is a very rare tumor of the uterine cervix. Presence of a large amount of extracellular mucin resulting in the formation of mucous lakes with a relative paucity of neoplastic cells within them is called colloid (gelatinous) carcinoma. ${ }^{1-4}$ The classification and prognosis of colloid carcinoma is controversial. $^{1-4}$

In the literature, only two colloid carcinoma cases with immunohistochemical features have been reported. ${ }^{1,2}$ One of the cases was reported as pure colloid carcinoma, ${ }^{2}$ while the second case was reported as colloid carcinoma with endocervical type adenocarcinoma in situ component. $^{2}$

In this case report, we present the histopathologic and immunohistochemical features of the tumor.

\section{CASE REPORT}

A 51-year-old female was admitted for examination of anemia. She complained of easy fatigability and uterine bleeding for 5 months. She has been in menopause for 2 years and has no past medical history. Her laboratory test results were within normal range except for hemoglobin levels $(9.8 \mathrm{mg} / \mathrm{dL})$. There was no palpable mass upon gynecologic examination. Chest X-ray and gastroscopic evaluation were normal. Endometrial and endocervical samplings were performed. Fragments of adenocarcinoma were identified in the assessment of the materials obtained from endocervical curettage. In imaging studies, the cervix was surrounded by a lesion with a diameter of $5 \mathrm{~cm}$, flattening the cervix wall. In detailed imaging studies, there was no additional lesion in gastrointestinal tract or in any other location. Cervical adenocarcinoma was prediagnosed according to the results of frozen section. Total abdominal hysterectomy with bilateral oophorectomy and pelvic and para-aortic lymph node dissection was performed. The cervix was grossly surrounded by an irregular tumor with a diameter of $5 \mathrm{~cm}$, and the cervix wall was flattened. The cross-sectional area was gelatinous and some parts were covered with mucinous layer (Fig. 1). In addition, endo- 
metrial lesion and a 3-cm-sized cyst in left ovary were detected. The remaining parts of the specimen were grossly unremarkable.

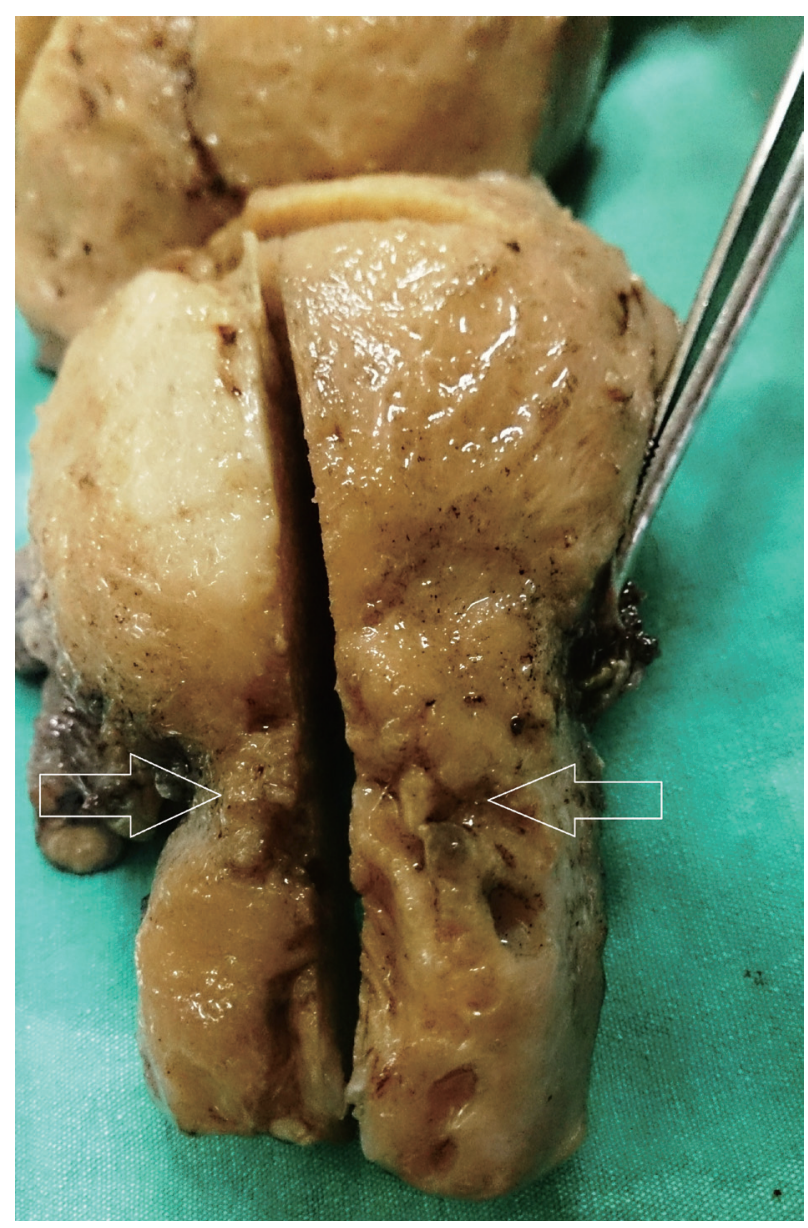

Fig. 1. Gross appearance of the tumor. The cervix is surrounded by an irregular tumor (arrows), with erosion in the mucosal surface covered by mucinous layer.
Formalin-fixed paraffin embedded tissue blocks of the surgical specimens were cut, deparaffinized and rehydrated. Sections were stained with hematoxylin and eosin. Immunohistochemistry was performed using manual polymer detection system with citrate buffer heat induced epitope retrieval. The following prediluted ready to use primary antibodies were used: MUC2 (M53, mouse monoclonal antibody, Thermo Fisher Scientific, Waltham, MA, USA), MUC5AC (USM1, mouse monoclonal antibody, Thermo Fisher Scientific), MUC6 (CLH 5, mouse monoclonal antibody, Thermo Fisher Scientific), cytokeratin (CK) 20 (Ks 20.8, mouse monoclonal antibody), carcinoembryonic antigen (rabbit polyclonal antibody, Thermo Fisher Scientific), CD10 (S6C6, mouse monoclonal antibody, Thermo Fisher Scientific), p16 (INK4, BioGenex, San Ramon, CA, USA). Periodic acid-Schiff (PAS) and alcian blue (AB) stains were performed.

On histopathological examination, the tumor deeply invaded the cervical wall (Fig. 2A). Also, adenocarcinoma in situ (AIS) of intestinal and usual types were detected in superficial areas. In these areas, the surface and glands were lined by tall columnar cells with pseudostratified nuclei containing coarse chromatin, and goblet cells were observed in some areas. In deeper regions, numerous variably sized mucous lakes were present. Cuboidal or low columnar neoplastic cells forming tubular or cribriform structures floated within the mucous lakes or were lining the inner surface. Some of the mucous lakes did not contain any cellular component. Neoplastic cells had enlarged oval nuclei without conspicuous nucleoli and large apical cytoplasm containing basophilic mucus. They resembled goblet cells. Since they were inside the mucous lakes, they were flattened because of the pressure. Mitotic figures were rarely observed. Lymphovascular invasion was not shown. Approximately $80 \%$ of the invasive

Table 1. Immunohistochemical and histochemical findings

\begin{tabular}{|c|c|c|c|c|c|}
\hline & \multirow{2}{*}{ Colloid carcinoma cells } & \multirow{2}{*}{ Mucous lakes } & \multicolumn{2}{|c|}{ Adenocarcinoma in situ cells } & \multirow{2}{*}{ Adenocarcinoma (usual) } \\
\hline & & & Intestinal & Usual & \\
\hline MUC2 & + & - & + & - & - \\
\hline MUC5AC & + & + & + & + & + \\
\hline MUC6 & - & - & Weak+ & - & - \\
\hline CDX2 & Focally+ & - & Focally+ & Focally+ & Focally + \\
\hline CK7 & + & - & + & + & + \\
\hline CK20 & - & - & - & - & - \\
\hline CEA & + & Focally + & + & - & - \\
\hline p16 & + & - & + & + & + \\
\hline PAS & + & + & + & - & - \\
\hline Alcian-blue & + & + & + & - & - \\
\hline Mucicarmine & + & + & + & Focally+ & Focally + \\
\hline
\end{tabular}

CK, cytokeratin; CEA, carcinoembryonicantigen; PAS, periodicacid-Schiff. 
component was composed of the colloid carcinoma, and other invasive component was formed by the usual type of endocervical adenocarcinoma. The latter component consisted of glands or cribriform structures lined by tumor cells with pseudostratified large nuclei containing coarse chromatin. Intracellular mucin was reduced, and mitosis was increased. The tumor was invading the endometrial surface. Invasion of the cystic tumor tissue consisting of mucinous component was found in the left ovary and tumor infiltration was found in the serosa of the right fimbrial end. The peritoneal washing was positive for tumor cells and the pelvic lymph node was also positive.

The results of histochemical and immunohistochemical studies are presented in Table 1. Mucous lakes and tumor cells of both colloid carcinoma and AIS intestinal type were positive for MUC5AC and negative for MUC6, and the tumor cells were positive for MUC2 but mucous lakes were negative for MUC2
(Fig. 2B-D). Mucous lakes and tumor cells of both colloid carcinoma and AIS intestinal type were positive for PAS, $\mathrm{AB}$, and mucicarmine stains.

The patient was treated with chemotherapy and radiotherapy. She had generalised tumor recurrence 1.5 years after the surgery.

Written informed consent was obtained and this manuscript was written in accordance with the principles of the Declaration of Helsinki.

\section{DISCUSSION}

Colloid adenocarcinoma of the cervix is a very rare tumor and its histopathologic classification is not clear. In the literature, only two cases were reported as colloid carcinoma of the cervix. ${ }^{1,2}$ It is a unique subtype due to the phenotype of contained mucus, and its prognosis is controversial. Mucinous adenocarcinoma is
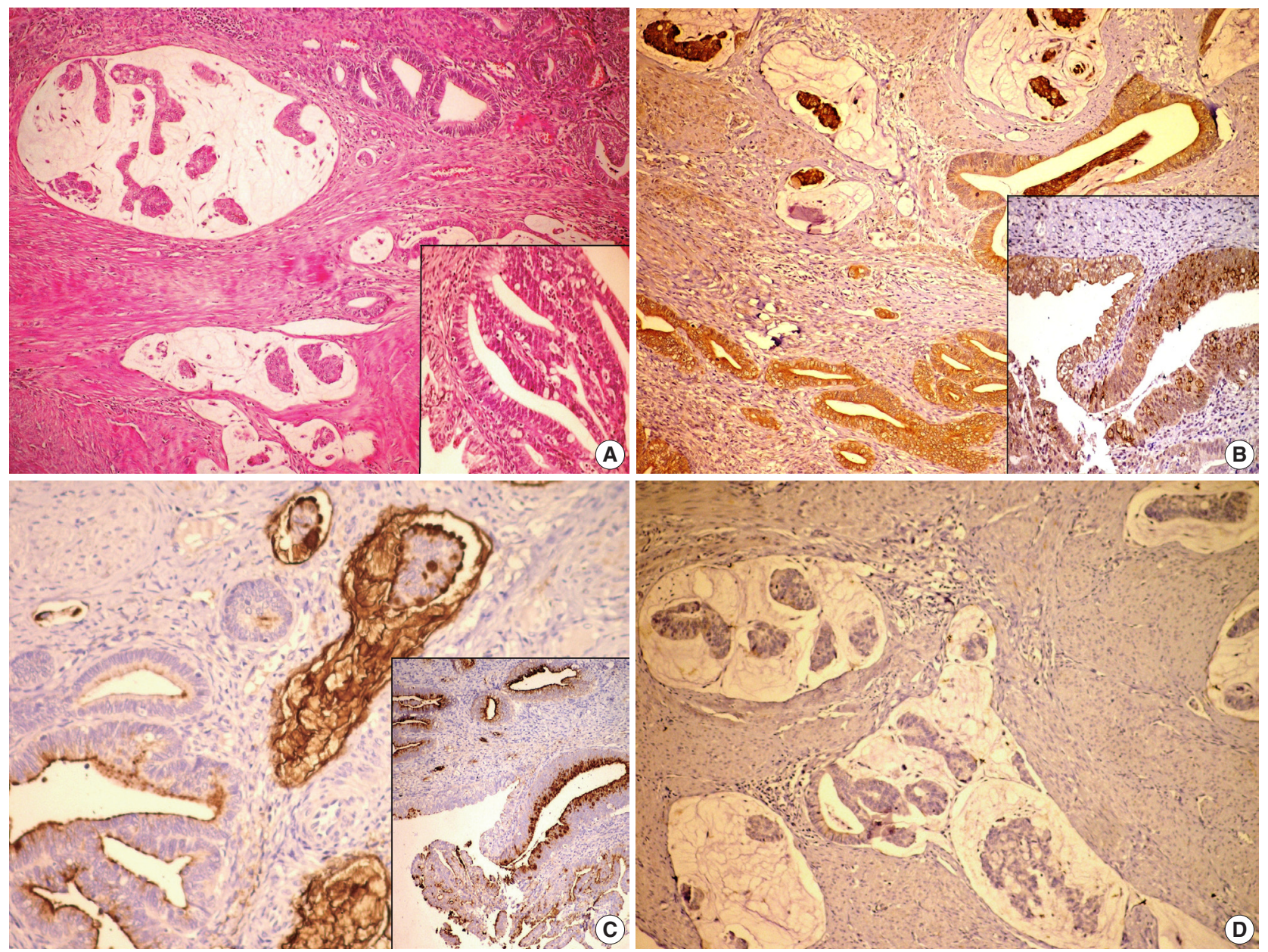

Fig. 2. Colloid carcinoma component is present in the deep area. Adenocarcinoma in situ (AIS), usual and intestinal types (inset), and endocervical adenocarcinoma (usual type) components are present in the superficial area (A). Colloid carcinoma cells and AlS intestinal type cells (inset) are positive for MUC2(B) and MUC5AC (C) and negative for MUC6 (D). 
classified into not otherwise indicated, gastric, intestinal, and signet-ring cell types according to the World Health Organization classification, ${ }^{5}$ but the classification of colloid carcinoma is controversial. It has been reported that large pools of mucin may occasionally be present within the stroma in endocervical adenocarcinoma usual type, and colloid carcinoma is treated and managed as a variant of the endocervical type. ${ }^{1}$ According to Young and Clement, ${ }^{6}$ mucinous adenocarcinoma consists of, in addition to the endocervical (usual type) adenocarcinoma, two variants: minimal deviation adenocarcinoma and intestinal type adenocarcinoma. Colloid carcinoma is a subgroup of the intestinal type. ${ }^{1,6}$ On the other hand, colloid tumor is also considered as a heterogeneous group of tumors which might have intestinal and endocervical phenotypes. ${ }^{1,2}$

The expression of $M U C$ genes is relatively tissue-specific. MUC5AC is a gastric foveolar epithelial type of mucin. MUC6 is a pyloric gland type of mucin and both types were expressed by endocervical cells while MUC2 is an intestinal type of mucin and not expressed by endocervical cells. ${ }^{1,27-9}$ When an epithelium undergoes malignant transformation, the mucin genes can undergo aberrant expression causing reduced production of an expected mucin and/or production of a structurally different and unexpected mucin within the transformed epithelium. ${ }^{8}$ Immunohistochemical analyses on different types of mucins have been conducted in pancreatic, gastrointestinal and endocervical tumors. While MUC2 is not expressed in normal endocervical epithelial cells, it is expressed in endocervical adenocarcinomas. ${ }^{2,8}$ It is observed that de novo MUC2 is expressed in neoplastic endocervical glands and postulated that MUC2 expression may accompany neoplastic transformation of endocervical glands. ${ }^{7}$ MUC2 staining pattern in our case is compatible with the staining of endocervical adenocarcinomas in the literature. ${ }^{7,8}$ Similar to our case, Shintaku et al. ${ }^{1}$ reported that while colloid cells stained with MUC2, mucin lakes did not. On the other hand, Ishida et al. ${ }^{2}$ did not refer to any staining. The difference in the staining pattern may be attributed to the mucin production during the malignant transformation and laboratory techniques. In our case, MUC5 AC staining was positive, which is compatible with the staining pattern of classical endocervical adenocarcinomas described in the literature. ${ }^{7,8}$ While Ishida et al. ${ }^{2}$ reported MUC5AC staining, Shintaku et al. ${ }^{1}$ did not refer to any staining.

In additon to the cervix, mucin expression in mucinous carcinomas of other organs is also discussed. ${ }^{9}$ In cases of colon carcinoma, MUC5AC and MUC2 stainings in mucinous and signetring cell carcinomas are detected at higher levels than in classical colon carcinomas. Also, it is considered that transformation process of the mucinous and signet ring cell carcinomas might be different from the traditional adenoma-carcinoma sequence of the colorectal carcinomas.?

CDX2 is a transcription factor expressed by the entire intestinal type of cervical adenocarcinomas and some conventional cervical adenocarcinomas. The importance of CDX2 lies in its essential role in the differentiation of the intestinal mucosal epithelium. ${ }^{10,11}$

In previously reported case of colloid carcinoma of the cervix, Shintaku et al. ${ }^{1}$ demonstrated that tumor cells were positive for MUC2 and CDX2, but negative for MUC5AC and MUC6. The authors concluded that the mucin-producing tumor cells were intestinal phenotype. ${ }^{1}$ In other case report of colloid carcinoma of the cervix, Ishida et al. ${ }^{2}$ found that MUC5AC and MUC6 were positive and MUC2 and CDX2 were negative in colloid carcinoma cells, and MUC5AC was positive and MUC2 and CDX2 were negative in endocervical type AIS component. In light of these evidences, the authors concluded that the mucinproducing neoplastic cells were endocervical phenotype, and that the endocervical type AIS can be a precursor lesion of colloid carcinoma. $^{2}$

In present case, the colloid carcinoma cells were positive for MUC5AC and MUC2, focal positive for CDX2, and negative for MUC6. Besides, in other parts of the tumor, AIS intestinal type revealed a similar staining pattern. We concluded that mucinproducing neoplastic cells were intestinal phenotype and intestinal type AIS might be a precursor lesion of colloid carcinoma.

Metastatic colloid adenocarcinoma from the gastrointestinal tract is an important tumor type in differential diagnosis of the colloid carcinoma of the cervix. In differential diagnosis, however, immunohistochemical staining alone is inadequate because the immunophenotyping of metastatic colloid adenocarcinoma from the gastrointestinal tract can be similar: positive for MUC2, MUC6, and CK 7 and positive/negative for CDX2 and CK20. ${ }^{2}$

In our case, immunohistochemical staining was not effective in the differential diagnosis of colloid carcinoma of the cervix from metastatic colloid adenocarcinoma arising from the gastrointestinal tract. Because there was no other tumor detected in the systemic examination of the patient, the presence of AIS was considered as an evidence for the primary cervical tumor.

In conclusion, since colloid carcinomas of the cervix are rare, detailed assessments with more samples are necessary to determine whether they are heterogeneous or homogeneous group depending on the type(s) of mucin and to describe the status of colloid carcinoma of the cervix in the classification of adenocarcinoma. 


\section{Conflicts of Interest}

No potential conflict of interest relevant to this article was reported.

\section{REFERENCES}

1. Shintaku M, Kushima R, Abiko K. Colloid carcinoma of the intestinal type in the uterine cervix: mucin immunohistochemistry. Pathol Int 2010; 60: 119-24.

2. Ishida M, Iwai M, Kagotani A, Yoshida K, Okabe H. Colloid carcinoma of the uterine cervix: a case report with respect to immunohistochemical analyses. Int J Gynecol Pathol 2014; 33: 248-52.

3. Walker AN, Mills SE. Unusual variants of uterine cervical carcinoma. Pathol Annu 1987; 22 Pt 1: 277-310.

4. Hurt WG, Silverberg SG, Frable WJ, Belgrad R, Crooks LD. Adenocarcinoma of the cervix: histopathologic and clinical features. Am J Obstet Gynecol 1977; 129: 304-15.

5. Kurman RJ, Carcangiu ML, Herrington CS, Young RH. WHO classification of tumors of female reproductive organs. Lyon: International Agency for Research on Cancer, 2014.
6. Young RH, Clement PB. Endocervical adenocarcinoma and its variants: their morphology and differential diagnosis. Histopathology 2002; 41: 185-207.

7. Riethdorf L, O'Connell JT, Riethdorf S, Cviko A, Crum CP. Differential expression of MUC2 and MUC5AC in benign and malignant glandular lesions of the cervix uteri. Virchows Arch 2000; 437: 365-71.

8. Baker AC, Eltoum I, Curry RO, et al. Mucinous expression in benign and neoplastic glandular lesions of the uterine cervix. Arch Pathol Lab Med 2006; 130: 1510-5.

9. Bu XD, Li N, Tian XQ, et al. Altered expression of MUC2 and MU$\mathrm{C} 5 \mathrm{AC}$ in progression of colorectal carcinoma. World J Gastroenterol 2010; 16: 4089-94.

10. McCluggage WG, Shah R, Connolly LE, McBride HA. Intestinaltype cervical adenocarcinoma in situ and adenocarcinoma exhibit a partial enteric immunophenotype with consistent expression of CDX2. Int J Gynecol Pathol 2008; 27: 92-100.

11. Sullivan LM, Smolkin ME, Frierson HF Jr, Galgano MT. Comprehensive evaluation of CDX2 in invasive cervical adenocarcinomas: immunopositivity in the absence of overt colorectal morphology. Am J Surg Pathol 2008; 32: 1608-12. 